Experimental and theoretical study of the Gouy phase anomaly of light in the focus of microlenses

This content has been downloaded from IOPscience. Please scroll down to see the full text. 2013 J. Opt. 15105708

(http://iopscience.iop.org/2040-8986/15/10/105708)

View the table of contents for this issue, or go to the journal homepage for more

Download details:

IP Address: 128.178.203.188

This content was downloaded on 23/09/2013 at 07:36

Please note that terms and conditions apply. 


\title{
Experimental and theoretical study of the Gouy phase anomaly of light in the focus of microlenses
}

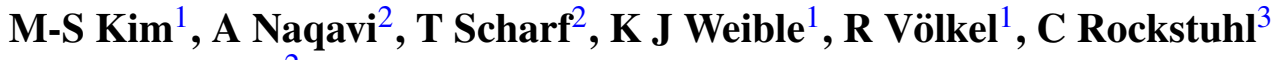 \\ and $\mathrm{H} P$ Herzig ${ }^{2}$ \\ ${ }^{1}$ SUSS MicroOptics SA, Rouges-Terres 61, Hauterive, CH 2068, Switzerland \\ 2 Optics and Photonics Technology Laboratory, Ecole Polytechnique Fédérale de Lausanne (EPFL), \\ Rue A-L Breguet 2, Neuchâtel, CH 2000, Switzerland \\ ${ }^{3}$ Institute of Condensed Matter Theory and Solid State Optics, Abbe Center of Photonics, \\ Friedrich-Schiller-Universität Jena, D-07743 Jena, Germany
}

E-mail:kim@suss.ch

Received 16 June 2013, accepted for publication 21 August 2013

Published 20 September 2013

Online at stacks.iop.org/JOpt/15/105708

\begin{abstract}
We report on the Gouy phase anomaly of light in the focus of cylindrical and spherical microlenses. The prime subject of our study concerns a discussion of how the very small size of microlenses affects the phase properties of light in their foci. We put emphasis on determining the amount of the Gouy phase shift for line and point foci within the limited axial space. Contrary to macroscopic lenses, the optical properties of microlenses are strongly governed by the effect of diffraction when their size tends to be comparable to the operation wavelength. In our study, we clearly show how such diffraction features affect the axial phase shift. For instance, phase singularities, which occur at discrete points on the optical axis where the total intensity vanishes for spherical microlenses, cause an additional axial phase shift when compared to the cylindrical microlens where those axial phase singularities are absent. The rotational symmetry of the Fresnel zones is the origin of such a difference between point and line foci.
\end{abstract}

Keywords: Gouy phase anomaly, microlens, micro-optics, longitudinal-differential interferometry

(Some figures may appear in colour only in the online journal)

\section{Introduction}

Light fields in the focal region of optical systems are of significant interest in many applications, not only for fundamental optical problems but also for advanced optical systems. In high-resolution optical systems for example, light is tightly focused where it interacts with a micro- or nano-size specimen. Particularly, it is known that the axial phase shift, e.g., the Gouy phase which occurs when light is focused [1-3], plays an important role in defining the optical response of many optical systems. Consequently, great attention led to profound studies in the past on the properties of focused light in general and on the Gouy phase in particular. The study of such anomalous phase features is of cardinal importance not just for conventional optical systems but also for micro- and nano-optical devices.

In general, the Gouy phase anomaly is characterized by $\alpha \cdot \pi / 2$ axial phase shift for a converging light wave passing through its focus upon propagation from minus infinity $(-\infty)$ to plus infinity $(+\infty)$. This Gouy phase is the additional phase advance the field experiences on axis when compared to a plane wave of the same frequency. The factor $\alpha$ is a dimension-related value, which equals 1 for a line focus, representing the 2D case (i.e., a cylindrical lens) and equals 2 for a point focus, representing the 3D case (i.e., a spherical lens). The physical meanings and the origin of this peculiar phase phenomenon continually induces discussions in which arguments are provided from different theoretical 
perspectives. For instance, an earlier study tended to seek an intuitive explanation of the phase anomaly based on the geometrical properties of Gaussian beams [4]. However, that approach is not sufficient to explain the $\pi / 2$ phase shift for a 2D focusing. Therefore, more advanced, sometimes sophisticated, theories were necessary to cover $2 \mathrm{D}$ and $3 \mathrm{D}$ focusing cases. Berry's geometrical (i.e., topological) phase, which is an additional geometric phase acquired by a system after a cyclic adiabatic evolution in parameter space, was applied to uncover its origin [5-8]. Even quantum mechanics has been considered to obtain insights into this anomalous effect [9-11]. More specifically, the study of Feng and Winful [10] relates Heisenberg's uncertainty principle to the Gouy phase with the spread in the transverse momenta. Since the Gouy phase exists for all waves, including sound waves, it is not necessary to introduce quantum mechanics to explain the physical insights and the origin of such a phase anomaly. Recently, the Gouy phase has been also explored in the context of more complicated wavefields, i.e., astigmatic wavefields, by theory [12] and by experiments [13]. There, the emergence is a natural consequence of two 2D focusing cases, such as sagittal and tangential foci. The 2D and 3D foci cases have been analyzed by applying corresponding mathematical functions that demonstrate different wavefront spacing for each focus case [14]. The Gouy phase anomaly has been intensively investigated for classical optical problems but also in new fields of science, such as the converging spherical terahertz $(\mathrm{THz})$ beam [15], matter waves [11], and photonic nanojets [16, 17]. However, for classical micro-optical focusing devices that generate a focal spot in free space, the Gouy phase has never been thoroughly discussed and we intend to close this gap with our study. Such devices represent a miniaturized version of Gouy's historical study $[1,2]$ where the mechanism of the light confinement mechanism is comparable, i.e. a spherical phase front is imprinted on an incident field that causes a light concentration due to constructive interference in the focal region. The mechanism that is used to explain the field confinement in such an optical focus is different when compared to other kinds of confined hotspots, such as e.g., photonic nanojets by the Mie scattering [16, 17], the spot of Arago by pure diffraction and interference of light [18], or the Talbot images by a grating's self-imaging effect [19].

The goal of our study is to demonstrate, for the first time, the Gouy phase anomaly of light in the focus of microlenses, whose size is smaller than $10 \mu \mathrm{m}$. By using cylindrical and spherical microlenses we intend to compare the observable Gouy phase to the anticipated values in more macroscopic optical systems -i.e. the well-known quantities of $\pi / 2$ for a line focus and $\pi$ for a point focus. Experimentally, we apply longitudinal-differential (LD) interferometry [18] to measure directly the phase anomaly in real-space. For theoretical verification of the measurements, we use the finite-difference time-domain (FDTD) method [20] that solves Maxwell's equations for the geometries of interest. This provides the means to compare our measurements to simulations. The remainder of this contribution is organized as follows. In section 2, the microlens and the measured geometrical parameters are briefly discussed. In section 3 , the details of the experimental setup and the numerical simulations are explained. The intensity and phase distributions near the foci of the spherical microlens array are presented and discussed in section 4. Section 5 is dedicated to the case of a cylindrical microlens, namely, a line focus (i.e., 2D focusing). In section 6, an overall discussion and summary of the Gouy phase of cylindrical and spherical microlenses will be given before conclusions are drawn in section 7 .

\section{Microlens conception and fabrication}

There are various types of micro-optical elements, refractive and diffractive. Among them, potentially the most popular and standardized element is the refractive microlens. Refractive microlenses find a variety of applications, including basic functions such as focusing and collimation. When compared to a conventional macro-sized lens, a microlens is usually defined as having a relatively small size, i.e. a diameter smaller than $1 \mathrm{~mm}$. However, the definition of the microlens or the microlens array is quite broad. For example, the International Organization for Standardization (ISO 14880-1, 2001) [21] says that a lens in an array with an aperture of less than a few millimeters, including lenses which work by refraction at the surface, refraction in the bulk of the substrate, diffraction or a combination thereof, is a microlens.

During the last two decades, a large number of fabrication techniques have been developed in order to achieve smaller and better micro-optical elements. One of the standard techniques is the micromachining process that uses photolithography followed by photoresist reflow and dry etching. It leads to plano-convex lenses and the fabrication processes require a substrate, usually a wafer, which the microlenses sit on [22]. There are equally several standard methods to characterize microlenses (see reviews in [23, 24]), such as a non-contact optical profiler, Twyman-Green interferometer and Mach-Zehnder interferometer. However, the characterization of small-sized microlenses is severely influenced by diffraction and scattering because of their low Fresnel numbers [25]. Moreover, the spatial resolution of the characterization system becomes a fundamental limitation. Recent progresses in micro- and nano-technologies allow better resolution for both measurement systems and fabrication systems. This boosts more precise and detailed investigations in micro- and nano-optical elements. For instance, recently, a $20-\mu \mathrm{m}$ diameter microlens has been fully characterized by multi-wavelength high-resolution interference microscope (HRIM) [26]. The surface shape, aberrations, and the 3D amplitude and phase field distributions near both the front and back foci have been investigated.

Such plano-convex microlenses are mainly applied to moderate or low numerical aperture (NA) applications. Aspherical microlenses (conic constant $k \neq 0$ ) are also obtainable by RIE-transfer of the reflow microlens into the wafer bulk material, typically fused silica or silicon. For the context of the present study, the Gouy phase of such a low-NA lens has to be considered along a relatively large propagation distance when compared to the lens size. This leads to 
Table 1. Geometrical parameters of the fabricated microlenses.

\begin{tabular}{lllllll}
\hline & Lens 1 & Lens 2 & Lens 3 & Lens 4 & Lens 5 & Lens 6 \\
\hline $\begin{array}{l}\text { Diameter or aperture } \\
\text { width }(\mu \mathrm{m})\end{array}$ & 5 & 6 & 7 & 8 & 9 & 6 \\
ROC $(\mu \mathrm{m})$ & 3.2 & 4.11 & 5.18 & 6.41 & 7.84 & 4.58 \\
Sag height $(\mu \mathrm{m})$ & 1.2 & 1.3 & 1.36 & 1.4 & 1.42 & 1.12 \\
Lens shape & Spherical & Spherical & Spherical & Spherical & Spherical & Cylindrical \\
\hline
\end{tabular}

difficulties in the experimental realization since an axial scan is required with a long distance but a sub-micron step size to make axial phase measurements at sufficient precision, i.e. the phase of the field has to be sampled sufficiently fine. To circumvent such problems associated with microlenses having a rather low NA, we designed for the observation of the Gouy phase in the foci of microlenses a compact experimental situation using small microlenses with a relatively high NA (e.g., NA > 0.5), that produce the focal spots close to the lens surface.

Therefore, in our study we use photolithography and the resist melting technique to fabricate a spherical microlens array on a glass substrate $(600-\mu \mathrm{m}$ thick 4 in glass wafer, $n=1.5)$. Note that for simplicity the dry etching process is excluded. Therefore, the lens material is the photoresist AZ1518 (Microchemical GmbH), whose refractive index after bleaching is 1.623 at $642 \mathrm{~nm}$ wavelength for our preparation procedure [27]. Figure 1(a) shows the 2D surface data of the $5 \times 5$ lens array, measured by confocal microscopy (NanoFocus AG). In each row, from left to right, the diameter of the microlenses increases from 5 to $9 \mu \mathrm{m}$ with increment of $1 \mu \mathrm{m}$. In order to check the production quality, five rows of the same lens array have been designed. The $1-\mu \mathrm{m}$ thick photoresist coating leads to the cylindrical patterns of the same thickness before the reflow process. The reflow causes different sag height for each diameter of the cylinder of the same thickness. We also prepared a cylindrical lens as shown in figure 1(b) and in this case a relatively long rectangular pattern with a width of $6 \mu \mathrm{m}$ has been reflowed, which leads to the sag of $1.12 \mu \mathrm{m}$. Note that the thickness of cylinder and rectangular patterns before reflow is the same, approximately $1 \mu \mathrm{m}$. Table 1 summarizes the geometrical parameters of the fabricated microlenses: the spherical lenses shown in the third row of figure 1(a) and the cylindrical lens in figure 1(b). The cylindrical lens is denoted as lens 6 .

\section{Experimental setup and procedure of numerical simulation}

High-resolution interference microscopy (HRIM) is a holographic microscopy in a sense that it records interferograms of an object with a reference field. By measuring multiple interferograms with an adjustable phase difference between object and reference field, the amplitude and phase distributions of the object field can be retrieved. In general, interferometric testing systems have been developed for two-dimensional measurements, for example, surface profiling and wavefront measurements of light fields in a plane of interest [28]. The major difference of our HRIM when

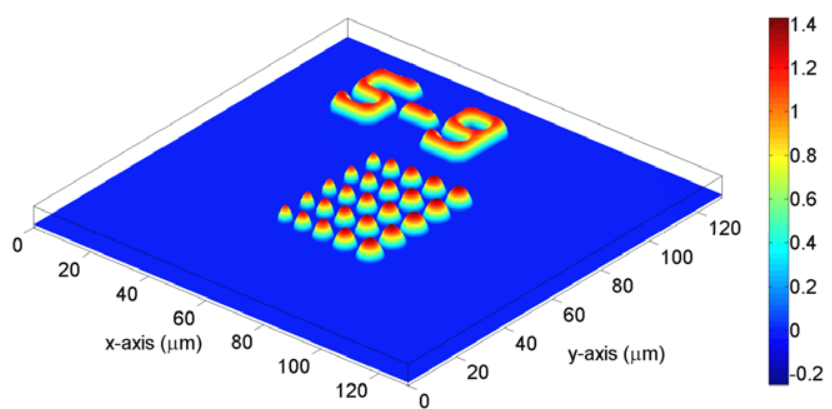

(a)

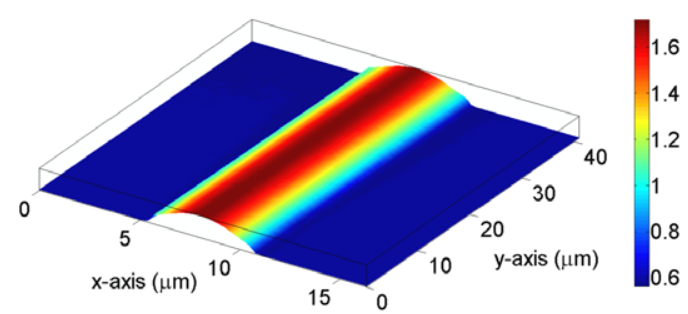

(b)

Figure 1. The measured 3D surface data of the fabricated microlens array using confocal microscopy: (a) spherical lenses and (b) a cylindrical lens. The color bar indicates the surface height in micrometers.

compared to other interference microscopes is the ability to record amplitude and phase fields in 3D by scanning the samples along the axial direction and repeating the measurement procedure. Furthermore, the HRIM is reinforced with auxiliary techniques known from conventional microscopy, which are often difficult to be implemented in other types of holographic or interferometric microscopes. The HRIM has been already proven as a powerful tool for the 3D characterization of macro-, micro-, and nano-optical elements. The HRIM operates in transmission with an in-line geometry by employing a Mach-Zehnder interferometer. A single-mode polarized laser diode is used for a light source (CrystaLaser, 642 nm: DL640-050-3). The first beam splitter (BS1) divides intensities to be sent in a reference and an object arm. In the reference arm, a piezo-electrically driven mirror is mounted to change the optical path length. The phase distribution of the wavefield is obtained by measuring the interference fringes at different mirror positions and employing a classical five-frame algorithm, which is called the Schwider-Hariharan method [29, 30]. In this scheme, five frames of the intensity pattern are recorded from which the spatially resolved transmitted phase is reconstructed, each frame being shifted by adding an optical path of $\lambda / 4$. 

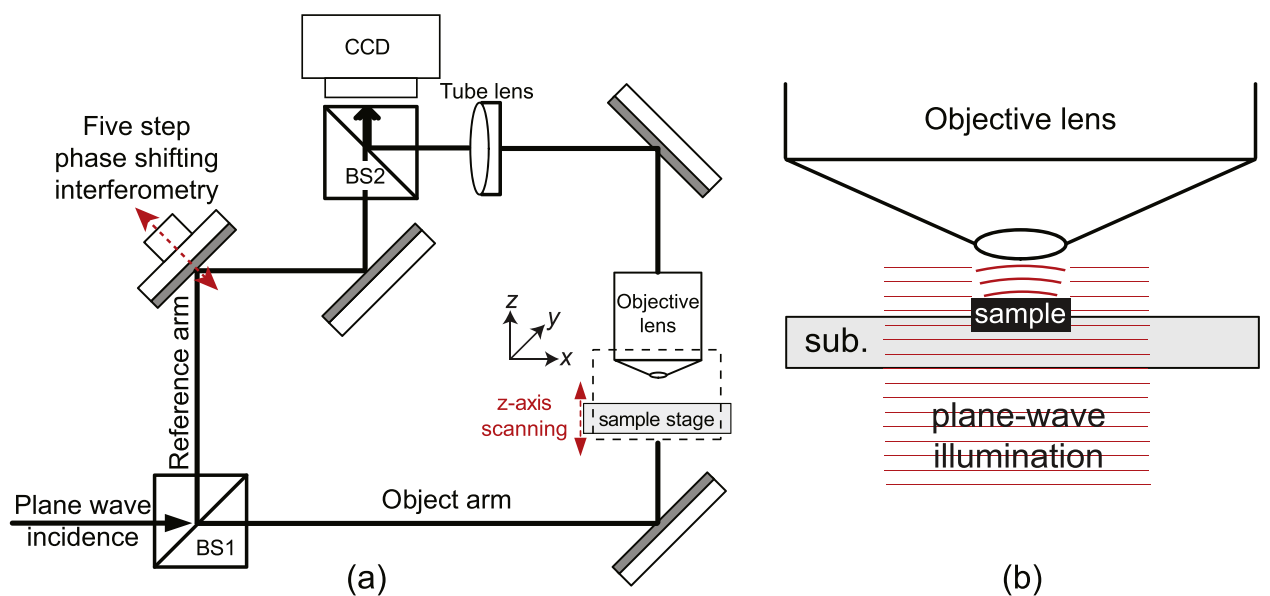

(a)

(b)

Figure 2. (a) Schematic of the longitudinal-differential interferometer based on the HRIM system. (b) A close-up illustration that shows the scanning situation near the sample, where the in situ self-reference wave (i.e., unperturbed incident plane wave) and the object wave (i.e., perturbed wave by the sample) are demonstrated. Scanning the sample along the $z$-axis leads to the 3D measurements.

Assuming that optical aberrations in the whole optical setup are negligible, the lateral resolution $d$ can be stated as the Abbe limit $(\Delta x)=\lambda / 2 \mathrm{NA}$. Therefore, the nominal lateral resolutions for the $100 \times /$ NA 0.9 objective (Leica Microsystems, HC PL FLUOTAR), which is equipped in the HRIM, is calculated to be $357 \mathrm{~nm}$ at a wavelength of $642 \mathrm{~nm}$. Along the optical axis, the Rayleigh criterion can be applied with the simplified formula derived as $\Delta z=\lambda n /\left(\mathrm{NA}^{2}\right)$, where $n$ is the refractive index of medium. The calculated axial resolution at a wavelength of $642 \mathrm{~nm}$ is $\Delta z=793 \mathrm{~nm}$. At $100 \times$ magnification, a pixel on our charge-coupled device (CCD) sensor (Sicon Corporation, CFW1312M camera with SONY ICX205AK image sensor of $1360 \times 1024$ pixels) corresponds to $46.5 \mathrm{~nm}$ in the object plane. This leads to the maximum field of view of the CCD camera of $64 \times$ $48 \mu \mathrm{m}^{2}$, which is sufficiently large to investigate the incident wavefront and the converging waves from microlenses. The overall experimental setup is described in detail elsewhere [26, 31, 32].

Longitudinal-differential (LD) interferometry [18] is based on the HRIM setup as a specific measurement mode. Its schematic is given in figure 2. Its purpose is essentially to directly measure the phase difference of the object field with respect to a referential plane wave, i.e. exactly the desired phase anomaly. The detailed measurement procedures can be found in the literature [18]. A certain fraction of the measurement domain needs to be reserved for an unperturbed wavefield to calibrate each measurement as shown in figure 2(b). In this domain the LD phase is just a trivial constant. This inclusion of the referential plane wave is necessary because of fluctuations in the long term stability. If the phase advance of the referential plane wave is added, the propagating phase can be retrieved.

To simulate the aforementioned structures, we used the MEEP software package [33], which is based on the finite-difference time-domain (FDTD) method [20]. The FDTD method is commonly used to provide full vectorial numerical solutions to Maxwell's equations and it can account for the near field as well as the far field simultaneously. We apply the periodic boundaries in the $x$ - and $y$-axes and the perfectly matched layer (PML) boundaries in the $z$-axis. For the 2D (i.e., cylindrical lens) and 3D (i.e., spherical lens) simulations, the grid of $400 \times 435(x \times z)$ and $400 \times 400 \times 330$ $(x \times y \times z)$ points are respectively used. The PML thickness is 50 grid points on the bottom and top of the simulation domain. A time step of 133 fs has been considered for the simulations. Stability of the FDTD simulation is guaranteed by considering a Courant factor of 0.5 . Thanks to the linearity of the problem, we used normalized parameters, i.e., $\mu_{0}=\varepsilon_{0}=c=1$. In the simulations, steady state is usually reached after a few thousands time steps. The simulations usually take $5-10 \mathrm{~h}$. For simplicity in simulations using the FDTD method, the NA of the measurement system is not considered. This NA of the measurement system filters out diffracted light associated with large tangential wavevectors. These angular components either propagate at increased angles or are associated with evanescent waves bound to the interface between glass and air. Generally, the finite NA of the imaging system prevents the collection of information on these fields in our far-field measurement system. However, this filtering turned out to be not necessary since the collecting NA of the system in each case is larger than the critical angular range into which light is scattered with a notable amplitude by the different considered lenses. Moreover, leaving the fields as they are not just makes it possible to observe in the numerical simulations fields in amplitude and phase as they are collected in our experiments, but also additional information such as the electromagnetic field distribution inside the lenses and near their surface can be provided. Since the natural outcome of the numerical simulations is the absolute phase map, the $\mathrm{LD}$ phase is easily retrieved by subtracting the phase of a referential plane wave, which is known analytically, from the phase of the total field.

\section{Intensity and phase distributions of spherical microlenses}

The field distributions emerging from microlenses of diameter from 5 to $8 \mu \mathrm{m}$ are measured by the HRIM. The illumination 


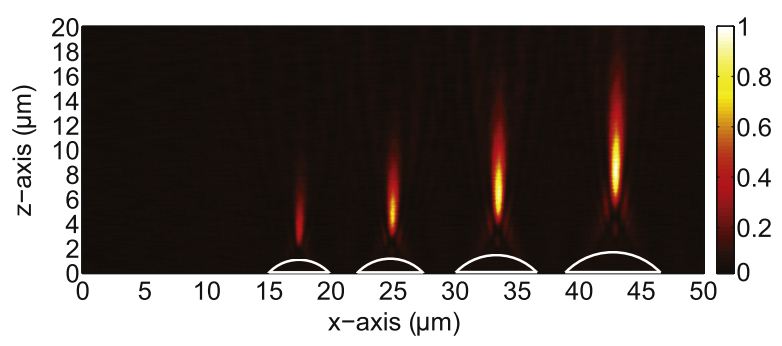

Figure 3. The measured $x-z$ intensity distribution. A plane wave of $\lambda=642 \mathrm{~nm}$ illuminates the microlenses of diameter $=5-8 \mu \mathrm{m}$ (from left to right), which propagates along the positive $z$-axis. Each lens focuses the incident light at different focal points where the peak intensity is proportionally increasing with the lens diameter. The region $x=0-15 \mu \mathrm{m}$ is where the in situ reference plane passes. The $z=0 \mu \mathrm{m}$ plane is the top surface of the substrate, on which the microlenses sit. The intensity is normalized to the maximal value.

is an $x$-polarized plane wave of $642 \mathrm{~nm}$ wavelength propagating along the positive $z$-axis and shines the lens from the substrate side. Figure 3 shows the measured intensity distributions in the $x-z$ plane, where one can observe the focal spot of each lens. The white spherical caps represent the microlenses. Since the focal length is extremely short, the back focus (i.e., the substrate side focus) stays in the substrate. Therefore, in this study, we investigate only the front focus, which stays in air. The magnitude of the intensity in each focal spot differs and the larger lens (i.e., 8- $\mu \mathrm{m}$ diameter) exhibits the focal spot with highest intensity because more light passes through the larger lens aperture.

LD interferometry requires an in situ reference plane wave, which is the unperturbed incident light passing aside of the sample. Therefore, we designed particular microlens array samples with a wide opening area to include the unperturbed illumination in the region of interest. In figure 3 , the $x=0-15 \mu \mathrm{m}$ is a region where the reference wave passes undisturbed. In order to image this large open area, the microlens array is shifted to the right side in the viewing field of the HRIM. This translation omits lens 5 (i.e., $9-\mu \mathrm{m}$ spherical lens) in the phase measurements. However, since there is no significant necessity for lens 5 , we concentrate on studying lenses 1-4 for the spherical lens and lens 6 for the cylindrical lens, which will be shown in section 5 . In experiments, the phase measurement originally leads to the LD phase map as shown in figure 4(a). The experimental errors due to vibration and the laser source instability can now be corrected by using the in situ reference plane wave that is present in the region of $x=0-15 \mu \mathrm{m}$ and which appears as a constant phase in the LD phase map. By wrapping this constant phase of the reference plane wave with a modulo of $2 \pi$, a propagation phase map, which is the counterpart of the simulated absolute phase map, is obtained and added in figure 4(b).

In figure 4, four microlenses show distinct phase distributions due to the different focal properties. In general, the light in the focal region demonstrates the shift from the converging wavefront to the diverging one. In the LD phase map, this change is shown as a triangular shape of a caustic-like phase feature flipping over. The merit of the
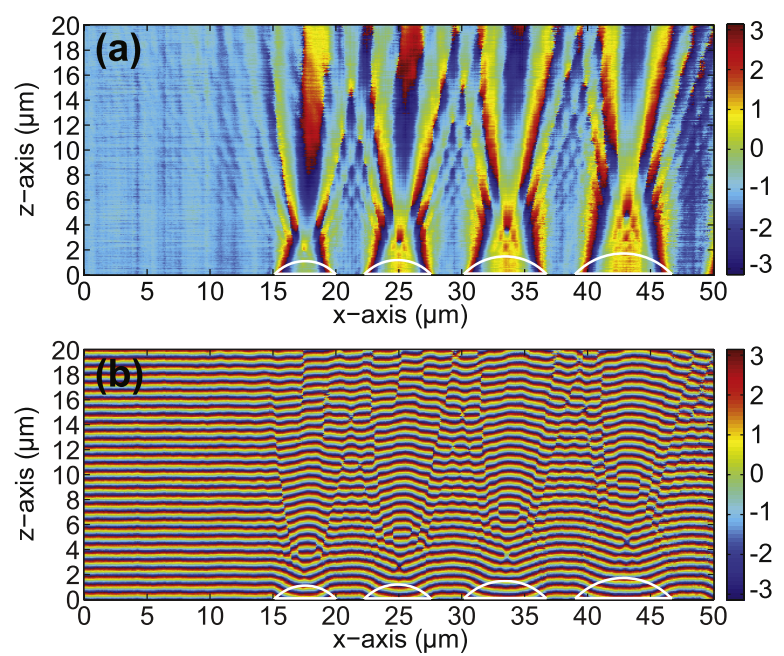

Figure 4. The measured $x-z$ phase distributions: (a) the longitudinal-differential phase map and (b) the propagation phase map. The reference plane wave passing the opening is shown in $x=0-15 \mu \mathrm{m}$ of both maps. Note that in the region of the reference plane wave the longitudinal-differential phase map is constant and independent on the propagation distance. In the remaining space, the figure shows the difference of the actual phase to the phase of that plane wave. The propagation phase is obtained by adding the phase advance of a plane wave in free space to each distance that can be calculated analytically according to $k_{z}$ and unwrapping it with a modulo of $2 \pi$. The color bar indicates the phase in radians.

propagation phase map is to directly visualize such wavefront shapes. Therefore, one can more straightforwardly identify spatial domains where such a change occurs.

Each column of figure 1(a) shows five microlenses of the same diameter. This setup was designed to verify the homogeneity of the fabrication and the corresponding optical response of the lenses. Since the Gouy phase of the 6- $\mu \mathrm{m}$ cylindrical lens will be compared with that of a spherical lens, the homogeneity of the $6-\mu \mathrm{m}$ spherical lens is of interest now. In order to achieve the same illumination conditions, the sample shown in figure 1 (a) is rotated $90^{\circ}$ in the clockwise direction. Figure 5 shows the $x-z$ intensity and phase distributions across four microlenses of the same diameter. For the referential plane wave, we move the lens array to the right side of figure 5. Thus, only four lenses are imaged. The reconstructed propagation phase map is shown as well. In this case, the four lenses show almost identical optical responses. Especially, when compared to figure 4(a), the LD phase map in figure 5(b) impressively demonstrates the same evolution of the axial phase emerging from each lens except for a negligible difference caused by measurement errors.

To verify the experimental results shown in figures 3-5, rigorous simulations using the FDTD method were carried out. Figure 6 shows the corresponding FDTD simulation results for the 5-8 $\mu \mathrm{m}$ lenses. The results show a good agreement with measurements shown in figures 3-5. In the intensity maps, just before the bright focal spot along the $z$-axis, a dark intensity region is found. It originates from the diffraction at the lens aperture. Such a dark intensity region is a typical demonstration of the destructive interference of diffracted light, where a phase singularity originates. It leads 

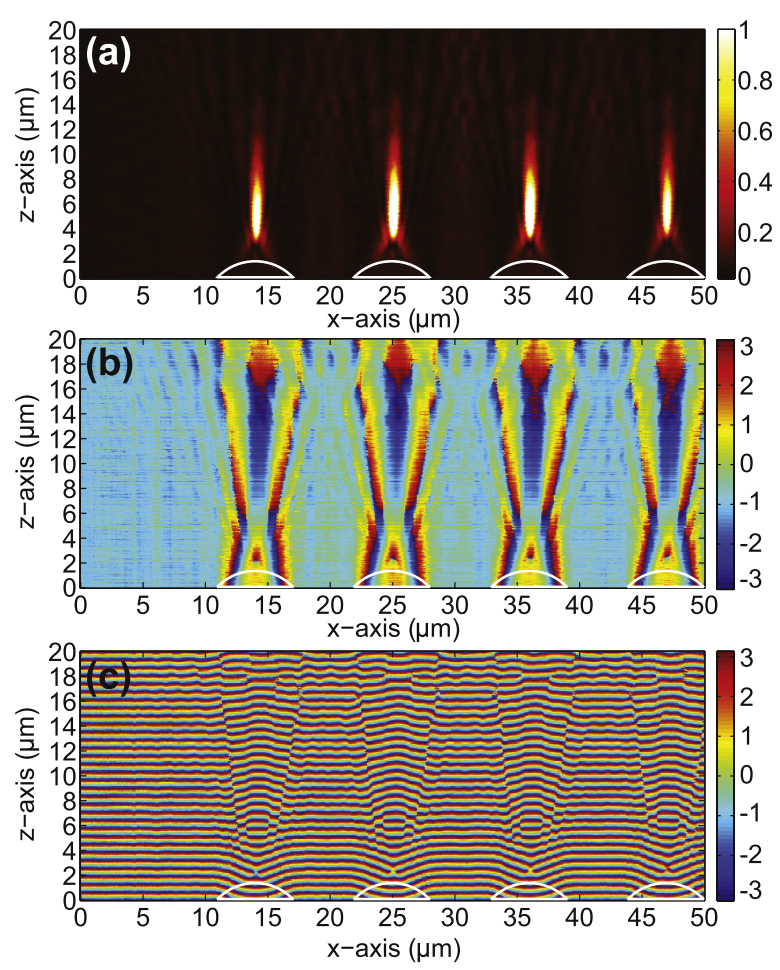

Figure 5. The measured $x-z$ intensity phase distributions of four spherical microlenses of diameter $=6 \mu \mathrm{m}$ : (a) the intensity, (b) the longitudinal-differential phase map and (c) the propagation phase map. The lenses are from the second column of figure 1(a) and the lens array is rotated $90^{\circ}$ in order to achieve the same illumination condition.

to a discontinuity of the wavefront (i.e., iso-phase line) in the absolute phase maps (see the region between the lens and the bright focal spot in the absolute phase maps of figure 6). In this region, the axial phase shift is very quickly deviating from the overall gradient of the Gouy phase (see inside the caustic-like phase feature in the LD phase maps). This diffraction feature enlarges the amount of the axial phase shift, particularly in the space between the lens and the focal spot. It is different from a conventional lens focusing, where such influences of the diffraction are not prominent. Those phenomena are well demonstrated in the measurements shown in figures 3-5. A more detailed discussion concerning the axial phase profiles of experimental and numerical results is in section 6 .

\section{Intensity and phase distributions of a cylindrical microlens}

In this section, we present the study of the $2 \mathrm{D}$ focusing case, i.e. $6-\mu \mathrm{m}$ cylindrical lens (see the details of lens 6 in table 1 ), as a comparison to the $3 \mathrm{D}$ focusing by a spherical lens. Lens 6 is illuminated with the same conditions-that is, a plane wave of $642 \mathrm{~nm}$ with $x$-polarization. Figure 7 shows the experimental results for the intensity and phase distributions in the $x-z$ plane. The corresponding FDTD simulation results are shown in figure 8. In this case, the numerical results show an excellent agreement with the experimental results.

Compared to the results of the $6-\mu \mathrm{m}$ spherical lens, the diffraction feature, which leads to additional phase shifts in the LD phase map and discontinuity in the absolute phase map, is not observed. Moreover, the intensity in the triangular region between the lens and the focal spot is prominently stronger than that of the spherical lens. We can find reasons in the classical diffraction problem. When the diffracted amplitude field is compared to the peak amplitude in the focal spot, the diffraction of the slit leads to a relatively higher magnitude when compared to the case of the circular aperture. The most interesting parameter is the overall Gouy phase anomaly. As anticipated, the gradient of the Gouy phase is lower than that of the spherical lens. It can be seen that the color variation in figures $7(\mathrm{~b})$ and $8(\mathrm{~b})$ is less than that of figure 5(b) for instance. The actual axial phase shift (i.e., the Gouy phase) value will be shown in section 6 and further compared with the 3D focusing case.

\section{Gouy phase anomalies}

The Gouy phase of a focused monochromatic field at an axial point is defined as the difference between the phase of the object field and that of a plane wave of the same wavelength [34]. In our study, the LD phase maps directly provide such information in a longitudinal plane, which contains the optical axis of each lens. In order to quantify the Gouy phase of the cylindrical and spherical microlenses, the axial phase profiles along the center of the lens from the LD phase maps are extracted and analyzed here. Since the LD phase map is still wrapped by $2 \pi$, one can obtain the actual axial phase shift along the optical axis by unwrapping the LD phase profile. In general, the amount of the Gouy phase is quantified for $\pm \infty$. In our case, the rather small size of the integrated device allows us to deviate from an evaluation of the Gouy phase from minus infinity to plus infinity (i.e. $\pm \infty$ ) since the focal plane is close to the lens plane, i.e. only a few micrometers away from the lens vertex. Therefore, we limit the region of interest to the range within the depth of focus (DOF), which we define here as the axial full width at half maximum (FWHM) of the intensity profile. In microscopic imaging systems, the measured light field through a scattering object can contain artifacts because of the aberrations in the imaging objective. Only the field measured from the sample to the free space would be considered as true information without particular errors. Therefore, the proposed method in [17] to differentiate diffraction effects from the Gouy effect is not rigorously correct and not directly applicable to our study. Since the additional diffraction features appear out of the focal region, by limiting the region of interest (ROI) one can minimize the influence of the diffraction features in the analysis of the Gouy phase, especially for the spherical lenses.

Figure 9 shows the extracted, measured and simulated axial phase profiles of figures 7(b) and 8(b) along the center of the cylindrical lens. Inside the lens (i.e. $z<1.4 \mu \mathrm{m}$ ), the simulation sustains complicated field distributions due to multiple reflections back and forth in the lens as well as additional scattering. In the measurement, such distributions are not detectable since they are not far-field features. Therefore, these regions should be not considered. Moreover, the increasing deviation between measurement and simulation 

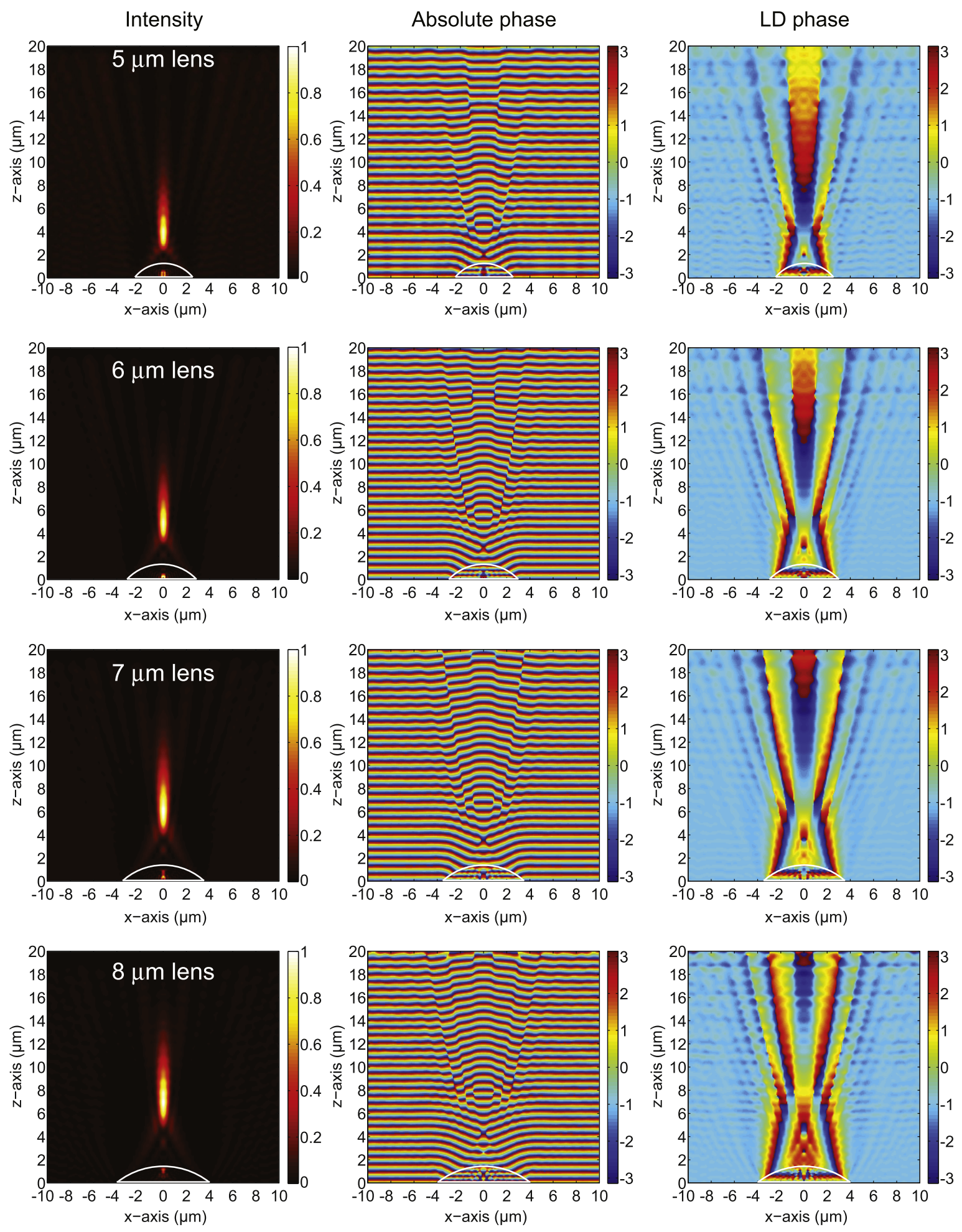

Figure 6. The FDTD simulation results for the $x-z$ intensity phase distributions of four spherical microlenses of diameter 5-8 $\mu \mathrm{m}$ (lens 1-lens 4), which correspond to the results shown in figures 3 and 4.

for excessive distances on the optical axis away from the lens, i.e. at $z>10 \mu \mathrm{m}$, is reminiscent of typical measurement errors. They are caused by an extremely low visibility of the interference fringe due the low intensity in this spatial domain. As a consequence, the measured phase becomes less reliable. In this spatial domain far away behind the focus practically no noticeable light can be found. Also, the scattered light emerging from the neighboring lenses is not considered in the simulation as a single lens is modeled. This could be an additional cause of deviation. Otherwise, the experimental result within the ROI is in excellent agreement with the simulation. The DOF is found in the intensity map of figure 8 

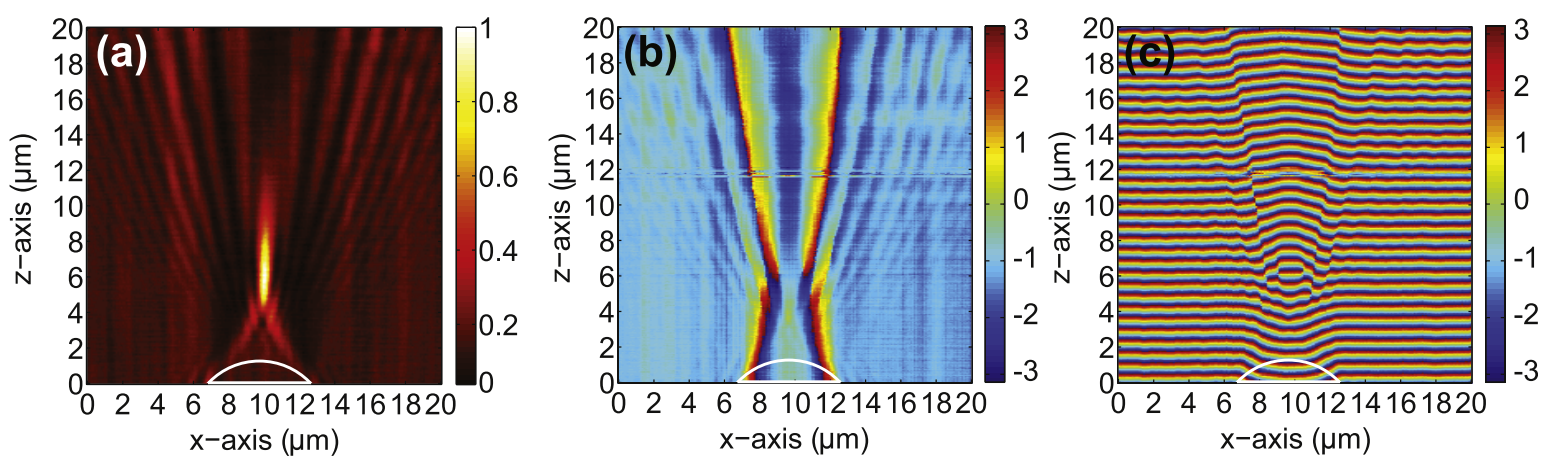

Figure 7. The measured $x-z$ intensity phase distributions emerging from a cylindrical microlens of aperture width $6 \mu \mathrm{m}$ : (a) the intensity, (b) the longitudinal-differential phase and (c) the propagation phase maps. The referential plane wave passes outside of the lens, $x=0$ to 7 and 13 to $20 \mu \mathrm{m}$. The intensity is normalized and the color bars in the phase distributions indicate the phase in radians.
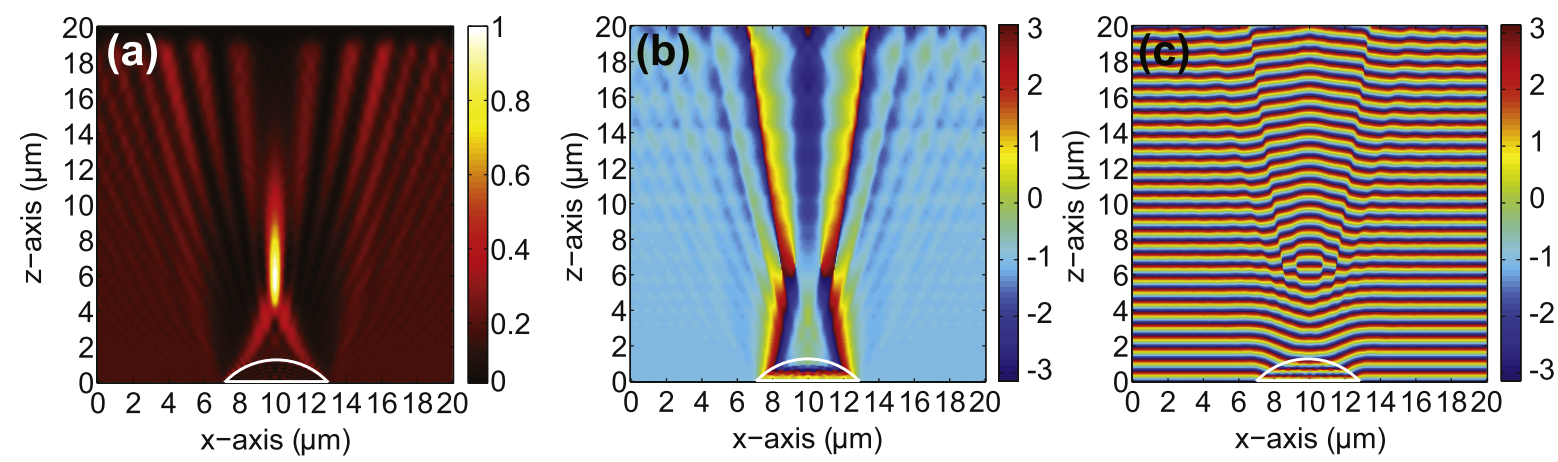

Figure 8. The FDTD simulation results corresponding to figure 7: (a) the intensity, (b) the longitudinal-differential phase and (c) the propagation phase maps. The intensity is normalized and the color bars in the phase distributions indicate the phase in radians.

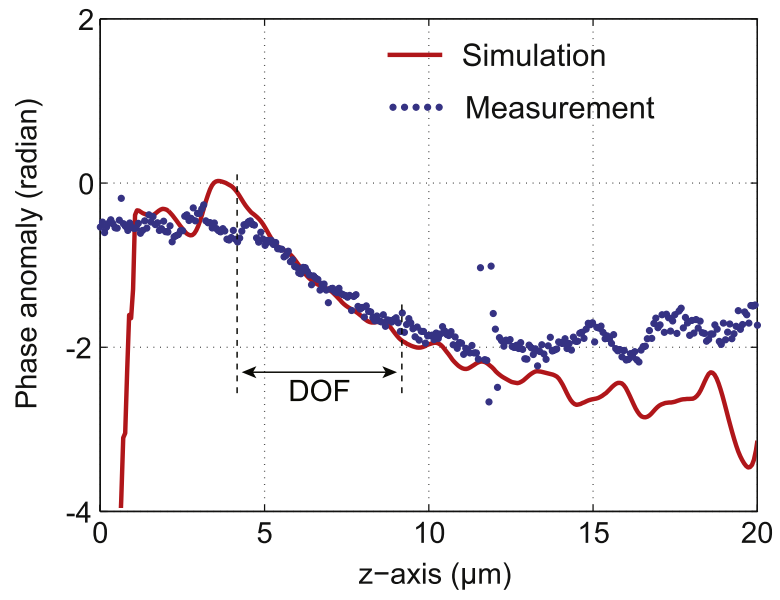

Figure 9. The Gouy phase of the cylindrical microlens of $6 \mu \mathrm{m}$ width: the extracted axial phase profiles from both experiment (figure 7) and simulation (figure 8). The depth of focus is $z=4.2-9.3 \mu \mathrm{m}$

to be in between $z=4.2$ and $9.3 \mu \mathrm{m}$. In this region, the axial phase shift equals $1.82 \mathrm{rad}(=0.58 \pi)$. This value corresponds to the Gouy phase of a cylindrical lens (i.e., 2D focus), which is quantified to be $0.5 \pi$ for $\pm \infty$.

Figure 10 shows the axial phase profiles for the $6 \mu \mathrm{m}$ spherical lens extracted from experiment and simulation. The experimental result is taken from the second left lens in figure 5(b) at $x=25 \mu \mathrm{m}$ and the numerical one from the second column of figure 6 . In this case, the experimental error shifted the measured phase profile by approximately $1 \mu \mathrm{m}$ in the negative $z$-direction. This is corrected in figure 10 by overlapping the profiles for better comparison. Inside the lens $(z=0-1.3 \mu \mathrm{m})$ and the region behind the focal spot $(z>10 \mu \mathrm{m})$, the discrepancy can be neglected as in the case of the cylindrical lens. The axial phase shift within the DOF $(z=3.6-6.9 \mu \mathrm{m})$ is found to be $2.71 \mathrm{rad}(=0.86 \pi)$. Although the relative difference from the anticipated value $\pi$ is larger than the relative deviation for the cylindrical lens, it still demonstrates that the 3D focus possesses approximately $50 \%$ larger Gouy phase that of a 2D focus within the axial range of the DOF. As discussed in section 4, for spherical lenses, the diffraction causes additional axial phase shifts between the lens and the focal spot (see the region $z=2-3.6 \mu \mathrm{m}$ ), where the dark amplitude spot leads to a pronounced phase evolution. Unlike the case of the cylindrical lens, there are zeros in intensity along the optical axis. The zeros for the 3D case (i.e., spherical lens) are a consequence of the fact that the Fresnel half-period zones are of equal area so that their total can exactly cancel [35]. The quantity of the axial phase shift is found to be approximately $6 \mathrm{rad}$, which is much larger than the fundamental Gouy phase of the focal spot. This is a special feature and situation, which can often be found in micro-optical problems. The Fresnel number $\left(=a^{2} / f \lambda\right.$, with $a=$ radius of the lens and $f=$ focal length) of such small microlenses is larger than unity. This simply indicates that 


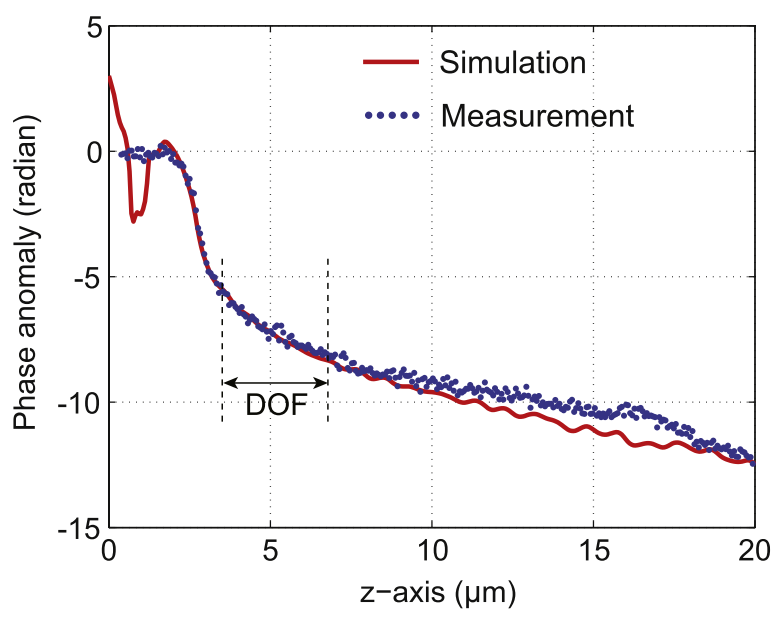

Figure 10. The Gouy phase of the spherical microlens of $6 \mu \mathrm{m}$ diameter: the extracted axial phase profiles from both experiment (figure 5) and simulation (figure 6). The depth of focus is $z=3.6-6.9 \mu \mathrm{m}$.

the Fresnel diffraction can represent the optical response of the lens. However, the optical regime falls into a special intermediate status because the Fresnel approximation, i.e., characteristic distance and size, $f$ and $a \gg \lambda$, is not valid for such small microlenses. Therefore, the criterion of the Fresnel number is inappropriate and rigorous treatments are necessary to properly understand the optical response and light interaction of such a small object.

\section{Conclusions}

The Gouy phase anomalies of 2D and 3D foci of smallsize microlenses have been investigated. The longitudinaldifferential interferometer plays a primary role in the investigation because it makes possible the direct measurement of the Gouy phase without any reconstruction process. The main interest is to quantify the amount of the Gouy phase for line and point foci happening in the micro-optical regime, in which the light field tends to demonstrate more complex evolution. Due to the small size of the test lens and the presence of the substrate in the test geometry, the nominal axial range from $-\infty$ to $+\infty$ for the Gouy phase was not applicable. In the LD interferometer, a reasonable measurement space starts from the substrate, on which the microlenses sit. Therefore, the region of interest has been set to be different from the conventional case-in our case, the ROI is the DOF of each lens.

When the size of the optical element becomes comparable to the operating wavelength, diffraction features significantly influence the light field distribution and its evolution. For example, particular phase features, such as e.g. spiral type phase singularities that occur in spatial domains where the intensity is zero at the selected point of the optical axis, are found in the 3D focus situation. The space between the lens and the focal spot possesses such dark spots due to destructive interference. The rotational symmetry of the 3D focus, in which the Fresnel half-period zones are of equal area so that their total amplitude can exactly cancel, is the origin of such dark spots. In this region where the dark spots result in the additional phase jumps due to the phase singularity, the phase evolution is relatively fast and large. This increases the axial phase shift to a much larger than the conventional Gouy phase of the point focus. Such dark amplitude points appear between the lens pupil and the focal spot; we can exclude axial phase jump originating from singularity by limiting the ROI to the vicinity of the focal spot (i.e., the DOF). Note that the line focus does not show such dark spots, and therefore there is no augmented axial phase shift due to the singularity.

Within the DOF, the 3D focus shows $0.86 \pi$ shift and the $2 \mathrm{D}$ focus results in $0.58 \pi$ shift. Although the $3 \mathrm{D}$ case does not yield twice the shift when compared to the 2D case, it demonstrates a relatively larger shift than the $2 \mathrm{D}$ case. This can be partially explained by the application of different axial ranges to quantify the Gouy phase when compared to the conventional case. The conventional values, such as $\pi$ for a point focus and $0.5 \pi$ for a line focus, are not expected. In the theoretical modeling, the NA filtering and influence of the neighboring lenses are not implemented, which might cause some minor deviations between experiments and simulations. Except for such negligible deviations, the experimental results show a good agreement with the FDTD simulations. This verifies the rigorous performance of the $\mathrm{LD}$ interferometer and our experimental design. Our results provide physical optical insights of wavelength-scale microlenses, whose optical regime is different from ray optics and pure far-field problems. When such small microlenses are applied in phase-sensitive problems, the Gouy phase and the additional increase of the axial phase shift should be taken into account. More attention is required for the point focus because the phase singularity along the optical axis speeds up the phase evolution and each dark spot augments an additional axial phase shift on top of the Gouy phase.

\section{Acknowledgment}

The research leading to these results has received funding from Swiss National Science Foundation under Project No. 200021_125177/1.

\section{References}

[1] Gouy L G 1890 C. R. Acad. Sci. 110 1251-3

[2] Gouy L G 1891 Ann. Chim. Phys. 24 145-213

[3] Born M and Wolf E 1999 Principles of Optics 7th edn (Cambridge: Cambridge University Press)

[4] Boyd R W 1980 J. Opt. Soc. Am. 70 877-80

[5] Simon R and Mukunda N 1993 Phys. Rev. Lett. 70 880-3

[6] Habraken S J M and Nienhuis G 2010 J. Math. Phys. 51082702

[7] Brand G F 2000 Int. J. Infrared Millim. Waves 21 505-18

[8] Subbarao D 1995 Opt. Lett. 20 2162-4

[9] Hariharan P and Robinson P A 1996 J. Mod. Opt. 43 219-21

[10] Feng S and Winful H G 2001 Opt. Lett. 26 485-7

[11] da Paz I G, Saldanha P L, Nemes M C and Peixoto de Faria J G 2011 New J. Phys. 13125005

[12] Visser T D and Wolf E 2010 Opt. Commun. 283 3371-5

[13] Rolland J P, Thompson K P, Lee K-S, Tamkin J, Schmid T and Wolf E 2012 Appl. Opt. 51 2902-8 
[14] Tyc T 2012 Opt. Lett. 37 924-6

[15] Wang X, Sun W, Cui Y, Ye J, Feng S and Zhang Y 2013 Opt. Express $212337-46$

[16] Kim M-S, Scharf T, Mühlig S, Rockstuhl C and Herzig H G 2011 Appl. Phys. Lett. 98191114

[17] Bon P, Rolly B, Bonod N, Wenger J, Stout B, Monneret S and Rigneault H 2012 Opt. Lett. 37 3531-3

[18] Kim M-S, Scharf T, Etrich C, Rockstuhl C and Herzig H G 2011 Opt. Lett. 37 305-7

[19] Kim M-S, Scharf T, Menzel C, Rockstuhl C and Herzig H G 2013 Opt. Express 21 1287-300

[20] Taflove A 1995 Computational Electrodynamics: The Finite-Difference Time-Domain Method (Boston, MA: Artech House)

[21] ISO 14880-1:2001 Optics and Photonics-Microlens Arrays-Part 1: Vocabulary

[22] Ottevaere H, Cox R, Herzig H P, Miyashita T, Naessens K, Taghizadeh M, Völkel R, Woo H J and Thienpont H 2006 J. Opt. A 8 S407-29
[23] Miyashita T 2005 Proc. SPIE 5858585802

[24] Miyashita T, Kato M and Ohta J 2009 Opt. Eng. 48073609

[25] Schwider J and Sickinger H 1997 Optik 107 26-34

[26] Kim M-S, Scharf T and Herzig H P 2010 Opt. Express 18 14319-29

[27] www.microchemicals.com

[28] Malacara D 2007 Optical Shop Testing 3rd edn (New York: Wiley) chapter 16

[29] Schwider J, Burow R, Elssner K-E, Grzanna J, Spolaczyk R and Merkel K 1983 Appl. Opt. 22 3421-32

[30] Hariharan P, Oreb B F and Eiju T 1987 Appl. Opt. 26 2504-6

[31] Kim M-S, Scharf T, Mühlig S, Rockstuhl C and Herzig H G 2011 Opt. Express 19 10206-20

[32] Kim M-S, Scharf T, Menzel C, Rockstuhl C and Herzig H G 2012 Opt. Express 20 4903-20

[33] http://ab-initio.mit.edu/wiki/index.php/Meep

[34] Pang X, Fischer D G and Visser T D 2012 J. Opt. Soc. Am. A 29 989-93

[35] Sheppard C J R 2013 Appl. Opt. 52 538-45 